\title{
Key governance principles underpinning urban sustainable development planning and management
}

\author{
J. Peris Blanes \\ Universidad Politécnica de Valencia, Spain
}

\begin{abstract}
Governance is assumed to be a core challenge for urban sustainability. Rather than a sectorial topic, urban sustainability is a cross cutting concern affecting and being affected - by many different interests and strategic urban issues. For this reason, urban sustainable development is mainly about how strategic decisions are made in the city, who is involved in these decision-making processes and how accountability is rendered.

In this article we propose a set of governance principles for programs on urban sustainability planning and management. They are derived from UNHABITAT experience in building capacities in cities around the world through two specialized programmes: Localizing Agenda 21 (LA21) and Sustainable Cities Programme (SCP).

Keywords: sustainable development, urban environment, governance, planning and management, local agenda 21.
\end{abstract}

\section{Introduction}

\subsection{Governance and urban sustainable development}

Urban sustainable development is a multidimensional concept involving environmental, economic, social and political issues [1,2]. These interlinks are so strong [3] that it is totally unfeasible to address them separately, so methodologies for urban sustainable development planning and management are called to include an integral and holistic vision leading to long-term, crosscutting and interdisciplinary approaches. 
In that sense, governance emerges as a relevant concept as it refers to the process whereby different actors interact in a multiple, complex and sometimes conflictive way for the definition of strategic issues [4] on urban sustainability. Governance captures complexity and dynamicity of processes [5], as well as power relations within them [6,7]. In particular, "participatory urban governance is about effective and collaborative planning, decision making and implementation to co-ordinate distinctive efforts of the local government, civil society organisations and the private sector towards the progressive attainment of sustainable urban development and local democracy" [8]. According to UNHABITAT the key ingredient to realizing the inclusive and sustainable city is "neither money nor technology, nor even expertise, but good urban governance" [9].

\subsection{The Localizing Agenda 21 and Sustainable Cities Programme}

The urban environment strategy of UN-HABITAT is mainly implemented through the Localizing Agenda 21 and the Sustainable Cities Programme which offer multi-year support to local authorities and their partners to locally contribute to the implementation of the Agenda 21 and the Habitat Agenda.

Despite minor differences in the approach, methodology and tools, both programmes share the overall philosophy on how urban sustainability should be addressed. On the main, they are capacity-building programmes aimed to strengthen competences of cities to address urban environment priority issues, while enabling replication and scaling-up of activities and mobilising anchoring institutions for support [10].

An emphasis is placed on the need for a shared vision to support the development and implementation of broad-based environmental action plans, focusing on context-specific aspects of the urban location and founded on a broad-based stakeholder participatory approach. Moreover, they enhance the capability of local authorities to integrate these action plans into strategic urban development plans, stimulating inter-sectoral synergy [11].

The programmes have been implemented since 1990 in more than 100 cities from 33 countries in Africa, Asia, Latin America and Eastern Europe. The cities are either medium-size or big. They are located in developing countries with a medium-low Human Development Index and an important role of international aid.

\subsection{The aim of the paper}

In this article we propose a set of governance principles for programs on urban sustainability planning and management which are derived from UN-HABITAT experience through Localizing Agenda 21 and Sustainable Cities Programmes.

Although described from a general perspective, the principles have a practical spirit and a clear normative purpose. They are expected to provide practitioners and academics with a general framework and a reference. The principles are like fundamental assumptions lying behind the overall philosophy of urban sustainability planning and management. The formulation of the principles has to 
be seen as a two ways learning process. On one hand they are based on UNHABITAT core values; on the other, they are built on lessons learned from real implementation in more than thirty countries from four different continents during more than fifteen years.

Specifically, the key governance principles proposed are the following ones:

1) Focus on connectivities, 2) Participation and stakeholders' involvement, 3) Building Partnerships and Networking, 4) Vision and action concurrency,

5) Learning oriented and Changing attitudes, 6) Pre-eminence of the local level, 7) Systematic Programme Monitoring and 8) Institutionalization.

A general description of each principle is given, including a justification and a critical assessment. In addition, some examples are provided.

\section{The key governance principles underpinning urban sustainable development planning and management}

\subsection{Focus on connectivities}

Urban sustainable development needs to be conceived as a complex mater involving environmental, economic, social and political dimensions that interact in a multiple, continuous and reciprocal way. This holistic and systemic vision suggests that, rather than tackling them separately, the focus should be on the way they interact. The goal is not reaching a static balance amongst environment, people and economy, but enabling win to win linkages amongst them.

It is all about promoting synergies and not reducing urban sustainability to a zero-sum game. Strategies involving different dimensions may lead to outcomes in which the aggregated result clearly exceeds what could be expected from separately dealing with them. For instance, environment may catalyse economic activity and promote people's commitment.

To this end, no matter how complex the situation may be that systemic vision and thinking are to be encouraged in order to truly allow synergies to emerge. Particularly, urban space has to be considered as the linking framework where environmental, social, economic and political interactions take place. For that reason, space is addressed as a major resource for urban sustainable development, not only from a land management perspective but by emphasizing the potentialities of spatial and built contexts in producing these balanced relationships.

For that reason, urban sustainability planning and management shifts from traditional sectorial approaches and goes forward to an over-riding concern with cross-cutting issues. That way, rather than only addressing environmental topics -such as air pollution or green areas- it aims to mainstream them in the overall urban planning process while emphasising the importance of connectivities between issues, sectors and institutions.

\subsubsection{Example 1: Community based waste management in Vinh City}

Localizing Agenda 21 programme at Vihn City led to implementation of an urban solid waste management project in several city neighbourhoods. Before 
the programme started, the solid waste collection activity only covered $35 \%$ of the city, so irregular waste dumping and traditional "burn-and-bury" were common practices. The project introduced new concepts for community based activities. An institutional framework was defined regarding the role of each stakeholder involved (ward authorities, block leader, waste collector, household...). Operational schemes were defined including ways and schedules of collection, required equipment and improvement of secondary collection points. In relation to political and social aspects, awareness rising campaigns were complemented with training courses leading to an active involvement of ward authorities and block leaders. Increase in service quality fostered people willingness to pay the sanitary fee, so new jobs were created for unemployed and poor people within the communities. In addition, unexpected reactions took place. As inhabitants experienced benefits, they were highly motivated to improve some public elements, as pavements and small inner-ward roads [12]. This way of tackling urban waste management as a matter of interrelated issues including cultural, organizational, economic and community involvement come out to be a key factor in the improvement of environmental quality of the communities in Vinh.

\subsection{Participation and stakeholders' involvement}

Interconnected issues demand integrative approaches, so the idea of dialogue is strongly supported as a core value of urban sustainability. This dialogue is expected to enable deliberation amongst three different types of stakeholders: state, civil society and private sector. Furthermore, to be effective it requires integrating three different levels: local, national and international [13].

Through this multiplicity of dialogues, urban environment planning and management relies on the knowledge, resources, legitimacy, power, authority and initiatives of different stakeholders. It is essentially a political decisionmaking process based on the understanding that tackling public problems in a shared-power world, needs collaboration of all those who are relevant to a particular problem [14].

With all these voices at stake the main priority becomes to agree on common priorities, so the possibility of building a consensus - or agreement - is the required condition for participatory processes to effectively contribute to urban sustainable development.

However, participation cannot be improvised. It needs to be properly structured, organized and coordinated. In that sense, participation is assumed to be more than a set of momentums, it is a continuous process in which city stakeholders not only need to be identified and mobilised, but also empowered through legislation and the required knowledge and capacities to effectively engage participation in an informed, constructive and sustained way.

\subsubsection{Example 2: Participation for Sustainable mobility in Bayamo}

The Localizing Agenda 21 project was launched at Bayamo in 2002. Participation of actors started with the Urban Environmental Profile, elaborated with contributions from more than 100 people or institutions. Widely 
disseminated, it served as the common information base for all stakeholders, enabling a more consistent and documented communication between them. Later on, more than 200 people attended the Urban Consultation and signed the Urban Pact, a public commitment for Bayamo sustainable development. Urban mobility was agreed as a priority [15] and a Working Group was created for listening different voices. After many never-ending meetings and truly strong negotiations, an agreement was reached between municipal authorities, police, private transport associations, informal sector representatives and community leaders. This led to some Demonstrations Projects which enabled joint formulation and implementation of initiatives. The success of the project in bringing people together to find innovative solutions has made it an example for replication in other Cuban cities.

\subsection{Building Partnerships and Networking}

In order to strengthen connectivities and participation on a continuous basis, partner relationships are to be encouraged through urban sustainability planning and management. This includes the establishment of cooperation links among local, regional and national authorities, local communities' organizations and other actors involved, particularly those with a major influence and concern, including the private sector.

The approach is founded on the belief that "sustainable planning is only possible, feasible and acceptable through networking. In such a context, all actors are treated as equal and an attempt is made to achieve a balance between private and collective interest" [13]. In fact, well-functioning networks are powerful instruments for promoting change due to "characteristics such as adaptability, flexibility, efficiency, promptness, creativity and contextual suitability" [13]. For that reason, the aim is not only to create new relations but to support and extend existing ones by assuming that a cultural change is required in order to make stakeholders co-responsible for the development and implementation of local sustainable policies. To this end, the most critical issue is the ability of partners to speak a common language, share interests and maintain a sense of reciprocity in mutually beneficial relations.

Finally, it is important to highlight that the strength of the programmes depends on the cooperation with supra-local institutions, so commitment of national and international institutions needs to be sustained for the achievement of long-term goals. Universities and Research Institutes can meaningfully contribute by creating networks of global support and knowledge management.

\subsubsection{Example 3: Networks-based capacity building strategy}

The capacity building strategy of UN-HABITAT is based on the engagement of a network of institutions (universities, training organisms, expertise focused NGO...) to provide sustained support and specialized knowledge to local authorities. Rather than a contractor-consultant relationship, involvement of institutions is a comprehensive process of mobilization, consultation, assessment of commitment and understanding of their partner role. Genuine interest and 
mutual understanding are needed to reach a long term commitment among programmes, institutions and cities [16].

\subsection{Vision and action concurrency}

Urban sustainable development planning and management incorporates a strategic approach combining long-term visions building with short-term solution-oriented actions taking [13].

Strategic thinking starts with a reflection on the deep nature of a project and its challenges. It is linked to the fundamental dilemmas of sustainable development and its limitations in a particular context. That way, vision formulation enables the city to define a shared and inspiring image of its future while defining key principles, long-term objectives, urban spatial structures and main issues of concern. This image serves as an appealing frame of reference that steers urban development.

Complementarily, action implementation is focused on the short and medium term and its scope is limited to specific goals and problem-solving. However, its interaction with strategic vision is important. It provides a space of action where specific ideas, strategies and principles are ground-tested while stakeholders, resources and commitments are mobilized enabling a more realistic decision making at strategic level.

These two different registers of urban planning "can together be understood as a (urban) project of projects" [13] in which visions and actions are in a continuous interaction. Provided that urban sustainability is not only complex but also rapidly changing and dynamic, visions should be adapted and up-dated in order to allow adjustments in strategies and ongoing implementations.

Urban sustainable development is bounded to a time framework that exceeds current horizons of political decision making. This induces a latent conflict among short-term visible results and long-term vision achievement. On one side, projects and products are important output of the process. On the other, capacity building, learning processes and partnership strengthening, although being less tangible are equally essential for long-term vision attainment [11].

\subsection{Learning oriented and changing attitudes}

Urban sustainable development needs to be seen as a complex affair with highly interrelated issues and a great diversity of perspectives and interests at stake. Therefore, simplifications must be avoided as there are not "universal" solutions to be applied. Even technical or expertise knowledge is not enough to tackle with diversity of perceptions and interests. Urban development requires new and innovative solutions, and these solutions should be elaborated in each particular context.

For that reason, the key issue of a planning process is to learn and develop new ways of doing things to achieve sustainable urban development. This means that the overall process is oriented towards "changing attitudes, stimulating new ways of thinking about issues and problems, developing new approaches to usual 
tasks and creating a willingness and determination to convert these innovations into lasting changes in operational practice" [17].

The basic idea is that actors mostly learn through practice and experimentation, and that actions have to be conceived so that people can learn more. It is not just the final results that count, but the whole learning-by-doing process itself, which requires a gradual but explicit desire to learn by all the partners involved. In consequence, the most effective way of promoting collective learning leading to attitudinal changes is through development of joint initiatives where stakeholders and citizens are able to participate in decision making and implementation.

Within this framework, learning processes are not primarily referred to a given level of expert knowledge, but rather to tacit knowledge generated through the participatory and deliberative processes. Specifically, this knowledge does not only stand for a specific way of conceiving urban issues, but also for developing new skills and assuming new values leading to new behaviours, routines and norms. These learning processes are aimed to encourage attitudinal change on how urban sustainable development is addressed.

To this end, a systematic way of learning is required. Learning-by-doing is the required condition for meaningful learning, but in order to capitalize experiences - and previous mistakes - a significant amount of time and effort should be devoted to reflect on experience [13]. Documenting reflections and synthesizing conclusions is the best way of turning them into explicit knowledge to be shared within the city and other contexts. For that reason, involving academic and research institutions in the planning process may give it an added strength.

\subsubsection{Example 4: Accumulating knowledge in Dar es Salaam}

The Sustainable Cities Programme in Tanzania started in the capital, Dar es Salaam in 1992. One of the main success of the programme is how specific knowledge on urban development planning and management has been generated and how different stakeholders have experienced deep changes in the way of tackling environmental issues as a consequence of intensive learning processes stimulated by the programme activities. By engaging the process, professionals from local administration and other stakeholders have gained specific knowledge and skills. Involvement of the University College of Lands and Architectural Studies has been important, as the experience has informed education and training curricula. That way, accumulated experience continues to benefit local authorities, as well as central government and international donors. Through participation in consultation forums, working group activities and implementation of demonstration projects, stakeholders' attitudes towards environment and participation have been influenced and there is no going back to the techno-bureaucratic and prescriptive former planning model [18]. 


\subsection{Pre-eminence of the local level}

Urban sustainable development planning acknowledges and emphasizes the significance and uniqueness of the local level. In that way, "cities need to be understood as dynamic crossroads of local, national and transnational placemaking processes" [13], producing multiple and conflicting identities. For that reason, localizing urban development should not be "interpreted as the one-way translation of universal principles into local conditions" [13], but as a continuous dialogue among different levels.

Acknowledgment of the local significance implies that urban sustainable development approach should be adapted to local circumstances and demands. This means that there is no universal methodology to be applied everywhere so methods and tools should be customized and adapted.

Furthermore, local reality is not only unique, but also dynamic and subject to multiple interpretations. For that reason, sustainable development urban planning moves away from traditional centralised approaches where "experts" were expected to understand needs of communities and then make a detailed design of projects with a precise acquaintance of its overall social, economic and environmental consequences. On the contrary, a more people centred approach recognises the need to build the capacities of local communities to prioritise and design their own projects based on their own analysis of needs [13]. For that reason, methodologies should be flexible enough [19] to cope with emergent issues appearing during the overall lifetime of the project.

It is not only a matter of having different interests involved but also of epistemology so long as, according to experience, it is simply impossible to predict several years in advance how things will actually evolve. To consider that a programme can only start when all insights and skills are fully formed not only reduces flexibility, but also limits innovation [11].

\subsection{Systematic Programme Monitoring}

In tune with a learning oriented philosophy, urban sustainable development planning should embody the goal of evaluating experiences and documenting the knowledge produced through the process. In addition, from the managerial perspective and alongside its flexible implementation pattern, it is essential to have an effective system of monitoring and assessment of progress [11]. This is the only way to consistently asses the different options arising during the process and thus making adequate choices.

In coherence with the overall philosophy of the approach, monitoring and evaluation should not only be focused on assessing results and impact, but also on evaluating processes.

Concerning impact monitoring a set of indicators should be defined in relation to each specific dimension of urban sustainability: environment, economy, social issues and governance. That way, urban development trends can be tracked, enabling proper action for encouraging progresses and restraining draw backs. From the point of view of process monitoring the goal is to know how decisions are made and to qualify the processes that led to them. The 
concern is mainly about participatory issues and normally focuses on measuring progresses in stakeholders' involvement. Their aim is to improve the quality of the process itself, to ensure that the right people are involved, to identify and address weaknesses in the decision-making activities and to provide early warning on a process which is meant to be long term [17].

In order to increase efficiency and rigour, it is important to use locally owned mechanisms to evaluate impact and processes [11]. Although common sets of indicators have been developed, the overall monitoring system should be agreed with local stakeholders' points of view. Otherwise, it will not only be difficult to gather the required information, but also they won't be fully representative of local reality.

\subsection{Institutionalization}

Urban sustainability planning and management is all about changes, changing the ways in which people think about urban issues and changing the ways in which people and institutions behave. Institutionalization is about making those changes permanent, building them into habits, procedures, norms and routines. It is also about engagement of urban institutions, civic organizations and citizens into new ways of understanding the city.

Institutionalisation means that main ideas of urban sustainable development planning and management are absorbed and integrated into institutions and organizations of the city. It means that stakeholders develop new routines leading to new practices for sustainable development regardless of whether they are within the framework of a specific sustainable development project or not [17].

For that reason, institutionalization implies important changes at different levels ranging from values and behaviours of people, to organizational and structural changes [17].

\section{Conclusions}

Assuming the importance of governance processes for urban sustainable development planning and management, a set of principles has been defined. Rather than a management tool, they are conceived as a sort of reference to understand and assess to what extent real experiences in cities work according to the proposed philosophy.

Concerning the level of generality, they are broad enough to be applicable to any programme on urban sustainability planning and management, but they are also specific enough to provide a reference for action. In that sense, they are aimed to provide effective orientation and enable evaluation of processes.

The principles include some cross-cutting ideas - such as systemic linkages at the urban locus - which shape their overall definition and content. For that reason, they do not have to be seen as independent ideas but as strongly linked one to each other. This overall appreciation of their interrelationships is what can truly provide a valuable framework for understanding their philosophy. 


\section{Acknowledgement}

It is greatly appreciated the support and contributions of the personnel from the Localizing Agenda 21 and Sustainable Cities Programme in the writing of this paper; in particular during my staying in UN-HABITAT headquarters in Nairobi.

\section{References}

[1] Bárcena, I., Ibarra, P. \& Zubiaga M., (eds). Desarrollo sostenible: un concepto polémico, S. Editorial Universidad del País Vasco: Bilbao, 2000.

[2] Ibarra, P. \& Unceta K., (eds). Ensayos sobre el desarrollo humano, Icaria: Barcelona, 2001.

[3] Selman, P., \& Parker, J., Citizenship, Civicness and Social Capital in Local Agenda 21. Local Environment, 2 (2), pp. 171-184, 1985.

[4] Graham, J., Amos, B., \& Plumptre, T., Principles for good governance in the 21st century. Policy Brief (15), Institute on Governance, 2003.

[5] Kooiman, J., Gobernar en gobernanza. Instituciones y desarrollo (16), pp. 171-194, 2004.

[6] Prats, J., Gobernabilidad democrática para el desarrollo humano. Instituciones y desarrollo (10), pp. 103-148, 2001.

[7] Gaventa, J., Triumph, Deficit or Contestation? Deepening the 'Deepening Democracy' Debate. Working Paper (264), Institute of Development Studies, 2006.

[8] Pieterse, E., Participatory Urban Governance, UNCHS: Nairobi, 2000.

[9] UN-HABITAT. The Global Campaign on Urban Governance. Concept paper. UN-HABITAT: Nairobi, 2002.

[10] UN-HABITAT \& UNEP, The Sustainable Cities Programme 1990-2000, UN-HABITAT \& UNEP: Nairobi, 2001.

[11] Tuts, R., Urban poverty reduction through good urban governance: How can Local Agenda 21 initiatives contribute? Lessons learned from UNHABITAT's experience. International Conference on "Local Agenda 21 in Development Perspectives". Brussels, 2002.

[12] Tuan, B.A. \& Khai, D.V., Report of the impact evaluation of the community-based solid waste collection in the frame of Localizing Agenda 21 project in Vinh City, ENDA Vietnam, 2001.

[13] Loeckx, A., Shannon, K., Tuts, R. \& Verschure, H. (eds). Urban Trialogues. Visions_projects_co-productions. Localising Agenda 21. UNHABITAT and PGCHS, K.U. Leuven: Leuven, 2004.

[14] Bryson, M.J. \& Crosby, C.B., Leadership for the common good. Tackling public problems in a shared power world. Jossey-Bass Publishers: San Francisco, 1992.

[15] Asamblea Municipal del Poder Popular de Bayamo, Diagnóstico UrbanoAmbiental. Ciudad de Bayamo. Proyecto Agenda 21 Local. 2003

[16] UN-HABITAT, Urban institutions' interest and commitment is imperative for environmental planning and management (EPM) support to cities - A strategy to mobilise and sustain. Newsletter September, 2006. 
[17] UN-HABITAT \& UNEP (1999): The SCP Process Activitie. The SCP Source Book Series. Volumes 1 to 8. UN-HABITAT \& UNEP. Nairobi.

[18] UN-HABITAT \& UNEP (2005): The SCP in Tanzania 1992-2003. The SCP Documentation Series, Volume 2. UN-HABITAT \& UNEP. Nairobi.

[19] UN-HABITAT \& UNEP (2000): City Experiences in Improving the Urban Environment. A Snapshot of an evaluation of six city initiatives in Africa. UN-HABITAT \& UNEP. Nairobi. 